\title{
Caracterização do óleo de buriti produzido no Norte de Minas Gerais: parâmetros de qualidade, perfil de ácidos graxos e conteúdo de carotenoides
}

Characterization of buriti oil produced in northern region of Minas Gerais: quality parameters, fatty acid profile and carotenoids content

Caracterización del aceite de buriti producido en el norte de Minas Gerais: parámetros de calidad, perfil de ácidos grasos y contenido de carotenoides

Recebido: 10/03/2021 | Revisado: 16/03/2021 | Aceito: 19/03/2021 | Publicado: 27/03/2021

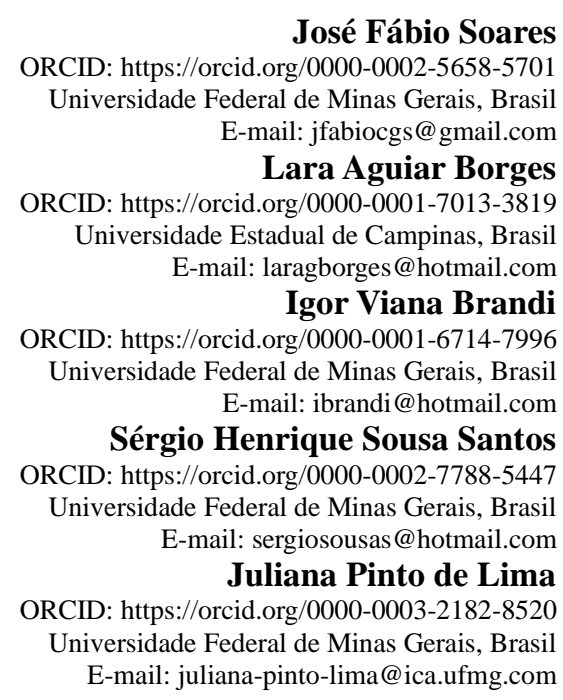

\section{Resumo}

O buriti (Mauritia flexuosa L.) é uma palmeira da família Arecaceae, que ocorre no Cerrado, sendo encontrado notadamente nas veredas. O fruto do buriti se destaca por possuir uma composição química complexa, com destaque para sua fração lipídica. Entretanto, dependendo da região de ocorrência dos frutos, este óleo vegetal pode apresentar características distintas. Nesse sentido, este trabalho teve como objetivo caracterizar o óleo de buriti proveniente de três municípios do Norte de Minas Gerais. Foram realizadas análises de caracterização físico-química, perfil de ácidos graxos por cromatografia gasosa, luteína, $\alpha$ - e $\beta$-carotenos por cromatografia líquida de alta eficiência e carotenoides totais por espectrofotometria. Os resultados demostraram que não houve diferença significativa entre as amostras de cada município analisado. Além disso, o óleo apresentou bom rendimento, ótima qualidade físico-química, além de ser uma fonte rica de ácidos graxos insaturados e carotenoides totais. Sendo assim, o óleo de buriti da região do Norte de Minas Gerais possui bom potencial para aplicação pela indústria de alimentos.

Palavras-chave: Cerrado; Mauritia flexuosa; Prensagem mecânica; Qualidade.

\begin{abstract}
Buriti (Mauritia flexuosa L.) is a palm of the Arecaceae family, which occurs in Cerrado, being found notably in the paths. Buriti fruit stands out for having a complex chemical composition, with na emphasis on its lipid fraction. However, depending of the region where the fruits occur, this vegetable oil may have different characteristics. In this sense, this work aimed to characterize the buriti oil from three municipalities in northern region of Minas Gerais. Physical-chemical characterization analyzes were performed, fatty acid profile by gas chromatografy, lutein, $\alpha$ - and $\beta$ carotenes by high performance liquid chromatography and total carotenoids by spectrophotometry. The results showed that there was no significant difference between the samples from each region analyzed. Besides, the oil presented good yield, excellent physical-chemical quality, in addition to being a rich source of unsaturated fatty acids and total carotenoids. Thus, buriti oil from the northern region of Minas Gerais has good potential for application by the food industry.
\end{abstract}

Keywords: Cerrado; Mauritia flexuosa; Mechanical pressing; Quality. 


\begin{abstract}
Resumen
El buriti (Mauritia flexuosa L.) es una palmera de la família Arecaceae, que se da en el Cerrado, encontrándose notablemente en los caminos. El fruto del buriti destaca por tener una composición química compleja, com énfasis en su fracción lipídica. Sin embargo, dependiendo de la región donde se produzca los frutos, este aceite vegetal puede tener diferentes características. En este sentido, este trabajo tuvo como objetivo caracterizar el aceite de buriti de tres municípios del norte de Minas Gerais. Se realizaron análisis de caracterización físico-química, perfil de ácidos grasos por cromatografía de gases, luteína, $\alpha$ - y $\beta$ - carotenes por cromatografía líquida de alta resolución y carotenoides totales por espectrofotometría. Los resultados mostraron que no hubo diferencia significativa entre las muestras de cada municipio analizado. Además, el aceite mostró buen rendimiento, excelent calidad físico-química, además de ser una rica fuente de ácidos grasos insaturados y carotenoides totales. Por lo tanto, el aceite de buriti de la región norte de Minas Gerais tiene un buen potencial de aplicación en la industria alimentaria.
\end{abstract}

Palabras clave: Cerrado; Mauritia flexuosa; Prensado mecânico; Calidad.

\title{
1. Introdução
}

O Cerrado Brasileiro é um dos biomas mais ricos no mundo, possuindo um patrimônio imensurável de recursos naturais renováveis, com destaque para as espécies frutíferas com características sensoriais intensas e singulares (Schiassi et al., 2018). Além disso, apresenta enorme diversidade sociocultural pela presença de comunidades tradicionais, que fazem uso dessas riquezas por meio do agroextrativismo sustentável, vivendo em sintonia com a natureza.

Uma das espécies nativas do Cerrado que merece destaque é o buriti (Mauritia flexuosa L.), uma palmeira da família Arecaceae (Freitas et al., 2017). Nesse bioma, o buriti é encontrado nas veredas, onde o solo é encharcado e úmido mesmo durante o período das secas. Os frutos da palmeira são compostos das seguintes partes: o pericarpo (ou casca) que possui escamas triangulares castanho-avermelhadas, o mesocarpo (polpa) é a parte comestível do fruto e, por fim, o endocarpo, um tecido esponjoso que recobre o caroço (Sampaio \& Carrazza, 2012). A polpa é a porção utilizada na agroindústria, sendo utilizada em diversos processos, como a produção de doces, sorvetes, geleias, vinhos (Sampaio \& Carrazza, 2012), inclusive na extração do óleo de coloração avermelhada, cada vez mais demandado por indústrias alimentícias e de cosméticos.

Recentemente, a riqueza química e eficiência terapêutica do óleo de buriti têm sido reportadas por alguns pesquisadores. Oliveira et al. (2020) relataram alto teor de ácidos graxos insaturados e carotenoides, além do efeito antioxidante e antidiabético em baixas concentrações do óleo. Ademais, Cruz et al. (2020) afirmaram que o óleo de buriti atua como imunomodulador contra Escherichia coli enteropatogênica. Por sua vez, Mansur et al. (2020) demonstraram que nanoemulsões contendo este óleo evita danos provocados por radiação ultravioleta por apresentar compostos com atividade fotoprotetora.

Sabe-se que a composição do óleo de buriti é muito complexa, podendo ser influenciada por diferentes fatores, tais como: estádio de maturação do fruto, região geográfica da produção, condições edafoclimáticas do local onde é produzido e também do tipo de manuseio utilizado (Santos et al., 2015; Pereira et al., 2018). Devido aos fatores interferentes, é possível encontrar óleos de buriti com características muito distintas. Rocha et al. (2017) afirmam que diferentes regiões de ocorrência dos frutos influenciam no teor dos compostos presentes no óleo, o que reforça a necessidade de evidências científicas para ampliar o conhecimento sobre as características do buriti.

Estudos relacionados à caracterização química do óleo de buriti proveniente da região Norte de Minas Gerais ainda são escassos, o que ressalta o desenvolvimento de pesquisas na área. Sendo assim, este trabalho teve como objetivo caracterizar o óleo de buriti produzido em três municípios do Norte de Minas Gerais, mais especificamente, determinar os parâmetros de qualidade, rendimento, perfil de ácidos graxos, bem como o teor de carotenoides. 


\section{Metodologia}

A metodologia utilizada neste estudo foi de natureza quantitativa por se tratar da coleta de dados numéricos por meio do uso de medições de grandezas, empregando-se métodos estatísticos para realizar previsões em relação às variáveis em estudo (Pereira et al., 2018).

\subsection{Matéria-prima}

Os frutos do buriti (Mauritia flexuosa L.) foram coletados em três municípios distintos, a saber: Brasília de Minas

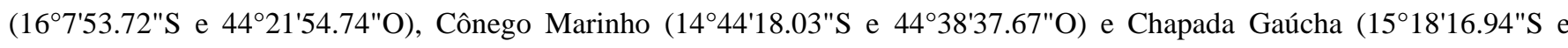
$\left.45^{\circ} 33^{\prime} 2.80^{\prime \prime} \mathrm{O}\right)$, todos pertencentes à região Norte do Estado de Minas Gerais, Brasil. Apenas frutos com ponto de maturação adequado, sem injúrias mecânicas, manchas e de tamanho uniforme foram selecionados.

Após a coleta, os frutos foram submetidos ao processo de higienização através da limpeza com água corrente e sanitização com solução clorada. Em seguida, os buritis foram submergidos em água potável durante 14 horas a fim de facilitar o desprendimento da casca. No despolpamento retiraram-se fatias da polpa, comumente chamadas de raspas, e estas foram colocadas em telas milimétricas e expostas ao sol para desidratação natural, processo semelhante ao realizado por agroextrativistas.

A polpa ficou em processo de secagem por 6 horas, atingindo uma umidade média de $11 \%$, sendo acondicionada em sacos plásticos de polietileno de baixa densidade (PEBD) de $15 \mathrm{~kg}$, com dimensões de $60 \mathrm{~cm}$ x $90 \mathrm{~cm}$, vedado, para evitar a absorção de umidade. Posteriormente as raspas foram encaminhadas para a indústria de múltiplos óleos (Cooperativa dos Agricultores Familiares e Agroextrativistas Grande Sertão LTDA) localizada em Montes Claros - Minas Gerais, onde realizouse a prensagem das amostras.

\subsection{Extração e obtenção do óleo bruto}

O óleo de buriti foi obtido por meio da prensagem mecânica em prensa tipo "Expeller". Para tanto, inicialmente pesou-se em balança digital (modelo 2124T, marca Mediza) $300 \mathrm{~kg}$ de polpa desidratada de buriti de cada região. Em seguida, a polpa foi submetida ao processo de trituração em Moinho de Facas (modelo MF32150, marca Scott Tech) para a redução da granulometria. As polpas trituradas foram colocadas diretamente na Prensa Mecânica tipo "Expeller" (modelo MPE-100, PI $10 \mathrm{CV}$, marca Ecirtec) para realizar o processo de extração do óleo, com controle de temperatura (máximo $50{ }^{\circ} \mathrm{C}$ ). Após a extração, o óleo ficou em processo de decantação durante 12 horas, seguindo para filtração em filtro prensa (Modelo FPE25/10 PI, marca Ecirtec), para retirada de todas as eventuais partículas presente no óleo. Em seguida, o óleo foi inertizado com a utilização de nitrogênio gasoso. Posteriormente, as amostras dos óleos de buriti foram recolhidas, acondicionadas em embalagem de $250 \mathrm{~mL}$ e armazenadas a temperatura ambiente $\left(25 \pm 2{ }^{\circ} \mathrm{C}\right)$ até o momento das análises.

\subsection{Rendimento}

O rendimento industrial do óleo de buriti obtido por prensagem a frio foi feito por diferença de massa, entre a massa da amostra inicial e a massa do óleo obtido. O resultado foi expresso em \%.

\subsection{Parâmetros de qualidade}

As análises de índices de acidez (mg KOH/g), peróxido (meq $\mathrm{O}_{2} / \mathrm{kg}$ ) e saponificação ( $\mathrm{mg} \mathrm{KOH} / \mathrm{g}$ ) foram realizadas de acordo com a metodologia descrita pela Official Methods of the American Oil Chemist's Society (AOCS, 2009). 


\subsection{Determinação do perfil de ácidos graxos}

Os ácidos graxos majoritários presentes no óleo de buriti foram identificados por meio de um Cromatógrafo Gasoso Capilar (modelo CGC, marca AgilenT 68650), equipado com detector de ionização de chama, injetor split e amostrador automático. Os compostos foram separados em coluna capilar de sílica fundida DB-23 (60 m x 0,25 mm x 0,25 $\mu \mathrm{m})$. A programação de temperatura da coluna foi iniciada em $110{ }^{\circ} \mathrm{C}$ por 5 minutos, aquecida gradativamente até $215{ }^{\circ} \mathrm{C}$. As temperaturas utilizadas no injetor e no detector foram 250 e $280^{\circ} \mathrm{C}$, respectivamente. As amostras foram injetadas no volume de 1,0 $\mu \mathrm{L}$, sendo o gás de arraste o hélio. Os ácidos graxos foram identificados pela comparação dos tempos de retenção de padrões puros de ésteres metílicos de ácidos graxos com os componentes separados das amostras e a quantificação foi feita por normalização de área $(\%)$.

\subsection{Determinação do perfil de carotenoides}

A extração de carotenoides nas amostras de óleo foi realizada de acordo com o protocolo proposto por RodriguezAmaya (2001) e a análise seguiu as condições cromatográficas desenvolvidas por Pinheiro-Sant'ana et al. (1998). Foi utilizado um sistema de Cromatografia Líquida de Alta Eficiência, com detector por arranjo de diodos (CLAE-DAD) com detecção a $450 \mathrm{~nm}$. A fase móvel - isocrática foi composta de metanol: acetato de etila: acetonitrila (80:10:10 v/v). O fluxo da fase móvel foi de $2.0 \mathrm{~mL} /$ minuto e tempo de corrida de 12 minutos. A coluna (Phenomene x Gemini, $250 \mathrm{x} 4 \mathrm{~mm}$ id, $5 \mu \mathrm{m}$ ) foi equipada com coluna guarda (Phenomenex ODS C-18, $4 \mathrm{~mm}$ x $3 \mathrm{~mm}$ ) (Phenomenex, Torrance, CA). A identificação dos carotenoides foi realizada através da comparação com os espectros dos padrões puros específicos (luteína, $\alpha$-caroteno e $\beta$-caroteno).

Os carotenoides totais foram extraídos e determinados de acordo com o procedimento descrito por Rodriguez-Amaya (2001). Os extratos foram diluídos em éter de petróleo, o espectrofotômetro utilizado foi o Thermo Scientific (Evolution 60S, USA) e a absorbância dos extratos foi medida a $449 \mathrm{~nm}$ usando coeficiente de absortividade molar de 2592. Os valores de carotenoides totais, em $\mathrm{mg} / 100 \mathrm{~g}$, foram calculados com base em uma curva padrão correlacionando a concentração de carotenoides totais (expressados em $\beta$-caroteno) e a absorbância da solução do pigmento utilizado.

\subsection{Análise estatística}

As determinações analíticas foram realizadas em triplicata $(n=3)$ e os resultados expressos como média aritmética \pm desvio padrão, sendo avaliados pela análise de variância (ANOVA) e as médias foram comparadas pelo teste de Tukey a $5 \%$ de probabilidade $(p<0,05)$, utilizando o software estatístico SISVAR 5.6.

\section{Resultados e Discussão}

\subsection{Rendimento do óleo}

Neste estudo foi observado, em média, um rendimento de $45 \%$ do óleo, considerando os cálculos com $300 \mathrm{~kg}$ de polpa desidratada. Este é um bom percentual, demonstrando que os frutos do buriti foram colhidos na época de maturação adequada. Em termos gerais, os processos industriais utilizados na extração de óleo vegetal consistem em prensagem hidráulica, prensagem mecânica contínua ou extração por solventes (Marques et al., 2015). Esta última é mais eficiente devido ao maior rendimento do óleo, porém pode gerar resíduos tóxicos no produto e no meio ambiente (Marques et al., 2015). Desta forma, a utilização de tecnologias limpas, como a prensagem mecânica, é recomendada porque não produz resíduos químicos, é sustentável, além de não alterar a estrutura do óleo.

Carvalho et al. (2011), ao estudarem o óleo de buriti proveniente da região Amazônica, encontraram um rendimento de óleo de 4,01\% pelo método artesanal, 21,5\% pelo método de prensagem hidráulica e 23,55\% para extração com solvente, resultados estes que mostram como pode ser variável o rendimento por diferentes processos de extração. O processo de 
extração do óleo em prensa tipo "Expeller" possibilita obter um maior rendimento por ser um equipamento industrial, quando utilizada à polpa desidratada (raspa), além de facilitar as operações de logística, transporte e melhorar os processos de estocagem do produto.

\subsection{Parâmetros de qualidade}

Conforme a Tabela 1, não foi observada diferença estatística $(p>0,05)$ entre os índices de acidez para os óleos obtidos dos diferentes municípios. Os resultados encontrados no presente estudo $(2,04-3,16 \mathrm{mg} \mathrm{KOH} / \mathrm{g})$ são adequados ao padrão de qualidade, o qual estabelece o limite máximo aceitável de 4,0 mg KOH/g para os óleos prensados a frio e não refinados, como é o caso do óleo de buriti (Brasil, 2005; Codex Alimentarius, 2017). A acidez indica a quantidade de ácidos graxos livres resultantes da decomposição dos lipídios (Vieira et al., 2018). Segundo Oliveira et al. (2020), a acidez é resultante do processo de rancificação do óleo durante a sua produção e o armazenamento, podendo provocar alterações nutricionais e sensoriais no produto. Desta forma, os óleos de buriti das três regiões estudadas apresentaram considerável estabilidade hidrolítica e oxidativa, sendo comprovada pelo índice de acidez que se enquadra no padrão de conformidade.

Tabela 1. Características físico-químicas dos óleos de buriti.

\begin{tabular}{lccc}
\hline Parâmetros & Brasília de Minas & Cônego Marinho & Chapada Gaúcha \\
\hline Índice de Acidez (mg KOH/g) & $2,64 \pm 0,72^{\text {a }}$ & $2,04 \pm 0,22^{\text {a }}$ & $3,16 \pm 0,28^{\text {a }}$ \\
Índice de Peróxido (meq $\left.\mathrm{O}_{2} / \mathrm{kg}\right)$ & $2,37 \pm 1,15^{\mathrm{a}}$ & $2,44 \pm 0,46^{\mathrm{a}}$ & $1,68 \pm 0,28^{\mathrm{a}}$ \\
Índice de Saponificação (mg KOH/g) & $193,36 \pm 0,11^{\mathrm{a}}$ & $193,23 \pm 0,03^{\mathrm{a}}$ & $193,29 \pm 0,06^{\mathrm{a}}$ \\
\hline
\end{tabular}

Valores expressos em média \pm desvio padrão $(n=3)$. Médias seguidas de letras iguais na mesma linha não diferem entre si pelo teste de Tukey $(p>0,05)$

Fonte: Autores.

Quanto ao índice de peróxido, os resultados médios obtidos foram 1,68 a 2,44 meq $\mathrm{O}_{2} / \mathrm{kg}$, sendo estatisticamente iguais $(p>0,05)$. O conteúdo de peróxido reflete a presença dos primeiros produtos formados durante o processo de oxidação dos óleos, conhecidos como hidroperóxidos. Em outras palavras, a quantidade de peróxidos indica o estado deteriorativo do produto, bem como a qualidade da matéria-prima utilizada. Conforme a Tabela 1, todos os valores determinados foram aproximadamente sete vezes menores do que o critério de aceitação, cujo limite máximo é 15 meq $\mathrm{O}_{2} / \mathrm{kg}$ (Brasil, 2005; Codex Alimentarius, 2017), denotando boa qualidade e bom estado de conservação dos óleos de buriti. De acordo com Siqueira et al. (2016), a extração a frio dos óleos vegetais não compromete a qualidade do produto e não causa processos oxidativos nos mesmos.

O índice de saponificação, por sua vez, expressa o tamanho da cadeia carbônica dos ácidos graxos presentes nos óleos vegetais (Pereira et al., 2019). Em outros termos, quanto maior este índice, menor a massa molecular dos ácidos graxos que compõe o produto (Ponte et al., 2017). Na presente pesquisa, os óleos de buriti não apresentaram diferença estatística significativa $(p>0,05)$ para o índice de saponificação e os resultados médios obtidos foram 193,23 a 193,36 mg KOH/g. No Brasil ainda não há legislação que determine esse parâmetro para o óleo de buriti, mas a Instrução Normativa $\mathrm{n}^{\circ} 49$ (Brasil, 2006) estabelece valores para índice de saponificação de óleos refinados, sendo o óleo de soja de 189 a 195 mg KOH/g, óleo de algodão de 189 a $198 \mathrm{mg} \mathrm{KOH/g,} \mathrm{milho} 187$ a $195 \mathrm{mg} \mathrm{KOH/g,} \mathrm{podendo} \mathrm{observar} \mathrm{que} \mathrm{o} \mathrm{óleo} \mathrm{de} \mathrm{buriti} \mathrm{possui} \mathrm{valores} \mathrm{entre}$ as faixas especificadas para outros óleos comerciais, podendo assim ser aplicado para fins alimentícios.

Embora as características físico-químicas dos óleos sofram variações de acordo com variedade da planta, condições edafoclimáticas e de pós-colheita, os índices de acidez, peróxido e saponificação das amostras avaliadas neste estudo 
corroboraram com outros trabalhos. Freitas et al. (2017), Pereira et al. (2018), Serra et al. (2019) e Oliveira et al. (2020), ao caracterizarem quimicamente o óleo de buriti, encontraram valores que variaram de 1,10 - 5,76 mg KOH/g, 2,23 - 12,05 meq $\mathrm{O}_{2} / \mathrm{kg}$ e 183,91- 203,39 mg KOH/g, respectivamente.

\subsection{Perfil de ácidos graxos}

Conforme a Tabela 2 verifica-se que os óleos apresentaram predominância de ácidos graxos insaturados (79,71 a $79,97 \%)$ e em menor proporção os ácidos graxos saturados (19,27 a 19,44\%). Dos ácidos graxos encontrados no óleo de buriti nas três regiões pesquisadas, o oleico (C18:1) representa o maior percentual com valores entre 77,32 a 77,82\%, seguido do palmítico (C16:0) com teores variando de 17,06 a 17,27\% e esteárico (C18:0) com valores de 2,17 a 2,21\%. Rocha et al. (2017) também relataram a predominância de ácido graxo oleico no óleo de buriti Norte Mineiro, com um percentual superior a 70\%. Em estudo realizado por Speranza et al. (2016), avaliando óleo de buriti da Amazônia Brasileira, os dois ácidos graxos de maior predominância foram os mesmos, sendo os valores próximos aos deste trabalho, 65,6\% para ácido oleico e 19,2\% para ácido palmítico. Estas variações nos resultados são previsíveis e evidencia como a composição química do óleo pode ser influenciada por características regionais e edafoclimáticas. Ademais, essas diferenças também podem ser atribuídas a outros fatores, como a maturação dos frutos, a etapa de secagem, bem como ao processo de extração e armazenamento do produto (Pereira et al., 2018).

Tabela 2. Perfil de ácidos graxos majoritários presente no óleo de buriti.

\begin{tabular}{lccc}
\hline Ácidos graxos (\%) & Brasília de Minas & Cônego Marinho & Chapada Gaúcha \\
\hline Palmítico (C16:0) & $17,17 \pm 0,41^{\mathrm{a}}$ & $17,06 \pm 0,07^{\mathrm{a}}$ & $17,27 \pm 0,12^{\mathrm{a}}$ \\
Esteárico (C18:0) & $2,21 \pm 0,09^{\mathrm{a}}$ & $2,21 \pm 0,06^{\mathrm{a}}$ & $2,17 \pm 0,09^{\mathrm{a}}$ \\
Oleico (C18:1) $-\omega 9$ & $77,32 \pm 0,50^{\mathrm{a}}$ & $77,82 \pm 0,03^{\mathrm{a}}$ & $77,51 \pm 0,11^{\mathrm{a}}$ \\
Linoleico (C18:2) $-\omega 6$ & $1,62 \pm 0,08^{\mathrm{a}}$ & $1,47 \pm 0,04^{\mathrm{a}}$ & $1,50 \pm 0,04^{\mathrm{a}}$ \\
Linolênico (C18:3) - $\omega 3$ & $0,77 \pm 0,02^{\mathrm{a}}$ & $0,68 \pm 0,04^{\mathrm{a}}$ & $0,74 \pm 0,03^{\mathrm{a}}$ \\
$\sum$ Ácidos graxos saturados & 19,38 & 19,27 & 19,44 \\
$\sum$ Ácidos graxos insaturados & 79,71 & 79,97 & 79,75 \\
\hline
\end{tabular}

Valores expressos em média \pm desvio padrão $(\mathrm{n}=3)$. Médias seguidas de letras iguais na mesma linha não diferem entre si pelo teste de Tukey $(p>0,05)$.

Fonte: Autores.

O perfil de ácios graxos presentes no óleo de buriti revela que este apresenta uma boa fonte de ácidos graxos monoinsaturados, representado pela grande fração de ácido oleico ( $\omega 9)$. De acordo com Pereira et al. (2018), há uma grande interesse em óleos que apresentam estes constituintes, pois a presença de ácidos graxos monoinsaturados na cadeia carbônica conferem uma preservação e estabilidade nas características dos produtos e, portanto, os tornam menos propensos a reações oxidaticas. Evidências científicas ainda demonstram que o óleo de oliva, comumente consumido pela população, é um produto muito estável em virtude do elevado teor de ácido oleico (63,12-79,68\%) (Borges et al., 2017). Ressalta-se, portanto, que o óleo de buriti é uma fonte tão rica em ácido oleico quanto o óleo de oliva, fato importante, pois este componente possui comprovado efeito benéfico à saúde. Altos teores de ácido oleico diminuem as concentrações dos triglicérides plasmáticos e da Low Density Lipoprotein cholesterol $(L D L$-c), contribuindo para a redução do risco de doenças cardiovasculares (Marcelino et al., 2019).

Aprofundando-se ainda nos ácidos graxos presentes nas amostras em estudo, constataram-se quantidades apreciáveis de ácido linoleico $(1,47-1,62 \%)$ e linolênico $(0,68-0,77 \%)$. Esses percentuais são interessantes, visto que são componentes 
considerados essenciais, isto é, não são produzidas no organismo humano. Os ácidos $\omega 6$ e $\omega 3$ atuam em inúmeros processos fisiológicos, a saber: saúde cognitiva, redução do risco cardiovascular, prevenção de doenças oculares, dentre outros (Arbex et al., 2015). Logo, o óleo de buriti é uma boa fonte de compostos responsáveis por benefícios à saúde.

\subsection{Perfil de carotenoides}

Conforme ilustrado na Tabela 3, não foi observada diferença estatística $(p>0,05)$ entre os valores de carotenoides totais, luteína e $\beta$-caroteno para os óleos provenientes do Norte de Minas Gerais. O conteúdo de carotenoides totais encontrado nos óleos de buriti $(562,85-582,62 \mathrm{mg} / 100 \mathrm{~g})$ foi bem maior do que o encontrado para os óleos de buriti oriundos de outras regiões (54,08-99,96 mg/100 g) (Santos et al., 2015; Freitas et al., 2017; Oliveira et al., 2020), muito provavelmente devido a variedade, grau de maturação, fatores agronômicos e processos de extração (Santos et al., 2015). Além disso, os resultados obtidos nesta pesquisa foram significativamente maiores do que o valor reportado para outros óleos comerciais, como o óleo virgem de palma (55,34 mg/100 g) e o óleo refinado de canola (0,0084 mg/100 g) (Mba et al., 2017). Segundo RodriguezAmaya et al. (2008), para um alimento ser considerado como fonte de carotenoides, é necessário ter um teor mínimo de 20 $\mu \mathrm{g} / \mathrm{g}$. Portando, observando os resultados apresentados para as amostras em estudo (Tabela 3), as mesmas apresentam mais de quinhentas vezes do valor recomendado por Rodriguez-Amaya et al. (2008), podendo ser consideradas fontes ricas desses constituintes alimentícios.

Tabela 3. Teor de carotenoides no óleo de buriti.

\begin{tabular}{lccc}
\hline Avaliação & Brasília de Minas & Cônego Marinho & Chapada Gaúcha \\
\hline Carotenoides totais $(\mathrm{mg} / 100 \mathrm{~g})$ & $577,53^{\mathrm{a}}$ & $562,85^{\mathrm{a}}$ & $582,62^{\mathrm{a}}$ \\
Luteína $(\mu \mathrm{g} / 100 \mathrm{~g})$ & $1,57^{\mathrm{a}}$ & $1,80^{\mathrm{a}}$ & $1,39^{\mathrm{a}}$ \\
$\beta$-caroteno $(\mathrm{mg} / 100 \mathrm{~g})$ & $206,47^{\mathrm{a}}$ & $324,63^{\mathrm{a}}$ & $201,25^{\mathrm{a}}$ \\
$\alpha$-caroteno $(\mathrm{mg} / 100 \mathrm{~g})$ & nd & nd & nd \\
\hline
\end{tabular}

Valores expressos em média \pm desvio padrão $(\mathrm{n}=3)$. Médias seguidas de letras iguais na mesma linha não diferem entre si pelo teste de Tukey $(p>0,05)$. nd: não detectado.

Fonte: Autores.

Os valores de luteína (1,39-1,80 $\mu \mathrm{g} / 100 \mathrm{~g})$ deste trabalho foram maiores em comparação aos óleos comerciais de canola e girassol, cujos valores não foram detectados na pesquisa de Flakelar et al. (2017). Contudo, o teor de luteína nos óleos de buriti foi menor que outros óleos brasileiros, tais como bacaba $(620 \mu \mathrm{g} / 100 \mathrm{~g})$, pupunha (1194 $\mu \mathrm{g} / 100 \mathrm{~g})$ e tucumã (4434 $\mu \mathrm{g} / 100 \mathrm{~g}$ ) (Santos et al., 2015). Tratando-se do $\beta$-caroteno, os óleos de buriti do Norte de Minas Gerais possuem quantidades bastante elevadas (201,25-324,63 mg/100 g) em comparação aos valores reportados em outras regiões (29,52-50,68 mg/100 g) (Santos et al., 2015; Freitas et al., 2017; Oliveira et al., 2020). Ademais, os valores de $\beta$-caroteno da presente pesquisa são significativamente superiores aos relatados em óleos comerciais de girassol $(0,14 \mathrm{mg} / 100 \mathrm{~g})$, canola (não detectado) e oliva $(0,31 \mathrm{mg} / 100 \mathrm{~g})$ (Flakelar et al., 2017). Quanto ao $\alpha$-caroteno, percebe-se que essa fração não foi detectada nas amostras em estudo (Tabela 3). Em contrapartida, Santos et al. (2015) encontraram um conteúdo de $\alpha$-caroteno de 1,92 mg/100 g ao analisarem a composição de carotenoides do óleo de buriti proveniente do Estado do Amapá.

Segundo Melendez-Martinez et al. (2013), as frações de $\beta$-caroteno e $\alpha$-caroteno apresentam atividade pró-vitamina A, ou seja, podem ser bioconvertidos em retinoides. Além disso, é importante destacar que os carotenoides participam de várias atividades fisiológicas, como o fortalecimento do sistema imunológico e a diminuição do risco de doenças degenerativas, como câncer, doenças cardiovasculares e outras (Rodriguez-Amaya et al., 2008). Portanto, o óleo de buriti norte-mineiro pode proporcionar diversos efeitos benefícios à saúde, devido ao seu elevado teor de carotenoides. 


\section{Considerações Finais}

O óleo de buriti obtido da região Norte de Minas Gerais possui bom rendimento quando extraído de polpa desidratada por meio de prensa mecânica contínua. Os resultados demonstraram que não houve diferença significativa entre os óleos obtidos dos três municípios avaliados, sendo que estes apresentaram qualidade físico-química em conformidade com as recomendações para óleos vegetais. Além disso, evidenciaram-se nas amostras altos teores de ácidos graxos insaturados (com destaque para o ácido oleico) e uma quantidade elevada de carotenoides totais, notadamente $\beta$-caroteno. Desta forma, o óleo de buriti da região do Norte de Minas Gerais possui potencial para uso na indústria de alimentos, podendo ser substituído parcialmente por óleos comerciais, ou mesmo para aplicação pela indústria farmacêutica ou cosmética.

Entretanto, sugere-se a realização de estudos sobre os efeitos do processamento e do tempo de armazenamento na qualidade do óleo de buriti a fim de verificar a estabilidade lipídica e dos compostos bioativos.

\section{Agradecimentos}

Os autores agradecem a Universidade Federal de Minas Gerais (UFMG), a Pró-Reitoria de Pesquisa (PRPq) da UFMG, a Cooperativa dos Agricultores Familiares e Agroextrativistas Grande Sertão LTDA, a Coordenação de Aperfeiçoamento de Pessoal de Nível Superior (CAPES), o Conselho Nacional de Desenvolvimento Científico e Tecnológico (CNPq) e a Fundação de Amparo à Pesquisa do Estado de Minas Gerais (FAPEMIG).

\section{Referências}

AOCS. (2009). Official methods and recommended practices of the American Oil Chemists' Society (6th ed.). Urbana, IL: USA.

Arbex, A. K., Bizarro, V. R., Santos, J. C. S., Araújo, L. M. M., Jesus, A. L. C., Fernandes, M. S. A., Salles, M. M., Rocha, D. R. T. W., \& Marcadenti, A. (2015). The impact of the essential fatty acids (EFA) in human health. Open Journal of Endocrine and Metabolic Diseases, 5, 98-104. http://dx.doi.org/10.4236/ojemd.2015.57013

Borges, T. H., Pereira, J. A., Cabrera-Vique, C., Lara, L., Oliveira, A. F., \& Seiquer, I. (2017). Characterization of Arbequina virgin olive oils produced in different regions of Brazil and Spain: Physicochemical properties, oxidative stability and fatty acids profile. Food Chemistry, 215, 454-462. https://doi.org/10.1016/j.foodchem.2016.07.162

Brasil. (2005). Resolução RDC $n^{o} 270$, de 22 de setembro de 2005. Regulamento Técnico Para Óleos Vegetais, Gorduras Vegetais e Creme Vegetal. Diário Oficial [da] União, Brasília, DF: Ministério da Saúde.

Brasil. (2006). Instrução Normativa $n^{\circ}$ 49, de 22 de dezembro de 2006. Aprova o Regulamento Técnico de Identidade e Qualidade dos Óleos Vegetais Refinados. Diário Oficial [da] União, Brasília, DF: Ministério da Agricultura, Pecuária e Abastecimento.

Carvalho, C. O., Scudeller, V. V., Sargentini Júnior, E., Fernandes, O. C. C., \& Bolson, M. A. (2011). Características físicas, químicas e rendimento do óleo de buriti (Maurita flexuosa L. f. - Arecaceae). BioTupé: Meio físico, diversidade biológica e sociocultural do Baixo Rio Negro. Manaus: INPA, $123-134$.

Codex Alimentarius. (2017). Codex standard for named vegetable oils. World Health Organization, Food and Agriculture Organization of the United Nations, Rome: Italy. Codex Stan 210-1999 (Amendment: 2005, 2011, 2013, 2015 and 2017).

Cruz, M. B., Oliveira, W. S., Araújo, R. L., França, A. C. H., \& Pertuzatti, P. B. (2020). Buriti (Mauritia Flexuosa L.) pulp oil as na immunomodulator against enteropathogenic Escherichia coli. Industrial Crops and Products, 149, 112330. https://doi.org/10.1016/j.indcrop.2020.112330

Flakelar, C. L, Prenzler, P. D., Luckett, D. J., Howitt, J. A., \& Doran, G. (2017). A rapid method for the simultaneous quantification of the major tocopherols, carotenoids, free and esterified sterols in canola (Brassica napus) oil using normal phase liquid chromatography. Food Chemistry, 214, 147-155. https://doi.org/10.1016/j.foodchem.2016.07.059

Freitas, M. L. F., Chisté, R. C., Polachini, T. C., Sardella, L. A. C. Z., Aranha, C. P. M., Ribeiro, A. P. B., \& Nicoletti, V. R. (2017). Quality characteristics and termal behavior of buriti (Mauritia flexuosa L.) oil. Grasas y Aceites, 68 (4), e220. http://dx.doi.org/10.3989/gya.0557171

Mansur, M. C. P. P. R., Campos, C., Vermelho, A. B., Nobrega, J., Boldrini, L. C., Balottin, L., Lage, C., Rosado, A. S., Ricci-Júnior, E., \& Santos, E. P. (2020). Photoprotective nanoemulsions containing microbial carotenoids and buriti oil: Efficacy and safety study. Arabian Journal of Chemistry, 13 (8), 67416752. https://doi.org/10.1016/j.arabjc.2020.06.028

Marcelino, G., Hiane, P. A., Freitas, K. C., Santana, L. F., Pott, A., Donadon, J. R., \& Guimarães, R. C. A. (2019). Effects of olive oil and its minor components on cardiovascular diseases, inflammation, and gut microbiota. Nutrients, 11 (8), 1826. https://doi.org/10.3390/nu11081826

Marques, F. G., Oliveira Neto, J. R., Cunha, L. C., Paula, J. R., \& Bara, M. T. F. (2015). Identification of terpenes and phytosterols in Dipteryx alata (baru) oil seeds obtained through pressing. Revista Brasileira de Farmacognosia, 25 (5), 522-525. https://doi.org/10.1016/j.bjp.2015.07.019 
Mba, O. I., Dumont, M. J., \& Ngadi, M. (2017). Thermostability and degradation kinetics of tocochromanols and carotenoids in palm oil, canola oil and their blends during deep-fat frying. LWT - Food Science and Technology, 82, 131-138. https://doi.org/10.1016/j.1wt.2017.04.027

Melendez-Martinez, A. J., Stinco, C. M., Liu, C., \& Wang, X.-D. (2013). A simple HPLC method for the comprehensive analysis of cis/trans (Z/E) geometrical isomers of carotenoids for nutritional studies. Food Chemistry, 138, 1341-1350. https://doi.org/10.1016/j.foodchem.2012.10.067

Oliveira, R. M. M., Pereira, F. T., Pereira, E. C., \& Mendonça, C. J. S. (2020). Óleo de buriti: Índice de qualidade nutricional e efeito antioxidante e antidiabético. Revista Virtual de Química, 12 (1), 2-12. https://doi.org/10.21577/1984-6835.20200002

Pereira, A. S., Shitsuka, D. M., Parreira, F. J., \& Shitsuka, R. (2018). Metodologia da Pesquisa Científica. UFSM.

Pereira, E., Ferreira, M. C., Sampaio, K. A., Grimaldi, R., Meirelles, A. J. A., \& Maximo, G. J. (2019). Physical properties of Amazonian fats and oils and their blends. Food Chemistry, 278, 208-215. https://doi.org/10.1016/j.foodchem.2018.11.016

Pereira, G. S., Freitas, P. M., Basso, S. L., Araújo, P. M., Santos, R. R., Araújo, P. M., Conde, C. F., Pereira, E. F. D., Haverroth, M., \& Amaral, A. M. F. (2018). Quality control of the buriti oil (Mauritia flexuosa L. f.) for use in 3-phase oil formulation for skin hydration. International Journal of Phytocosmetics and Natural Ingredients, 5(1), 1-5. https://doi.org/10.15171/ijpni.2018.01

Pinheiro-Sant'ana, H. M., Stringheta, P. C., Brandão, S. C. C., Páez, H. H., \& Queiróz, V. M. V. (1998). Evaluation of total carotenoids, alpha- and betacarotene in carrots (Daucus carota L.) during home processing. Food Science and Technology, 18 (1), 39-44. https://doi.org/10.1590/S010120611998000100009

Ponte, F. A. F., Rodrigues, J. S., Malveira, J. Q., Ramos Filho, J. A. S., \& Albuquerque, M. C. G. (2017). Avaliação físico-química dos óleos de babaçu (Orbignya speciosa) e coco (Cocos nucifera) com elevado índice de acidez e dos ácidos graxos (C6 a C16). Scientia Plena, 13 (8), 085301. https://doi.org/10.14808/sci.plena.2017.085301

Rocha, S. M., Rodrigues, M. T. O. S., Silva, D. S., Morais-Costa, F., Cardoso Filho, O., Nunes, Y. R. F., Arrudas, S. R., \& Fidêncio, P. H. (2017). Efeito do armazenamento nas propriedades físico-químicas do óleo de Mauritia flexuosa L. f (Arecaceae). Caderno de Ciências Agrárias, 9 (1), 31 -37.

Rodriguez-Amaya, D. B. (2001). A guide to carotenoid analysis in food. International Life Sciences Institute.

Rodriguez-Amaya, D. B., Kimura, M., \& Amaya-Farfan, J. (2008). Fontes Brasileiras de Carotenoides: tabela brasileira de composição de carotenoides em alimentos. MMA/SBF.

Sampaio, M. B., \& Carrazza, L. R. (2012). Manual tecnológico de aproveitamento integral do fruto e da folha do buriti (Mauritia flexuosa). Brasília - DF: Instituto Sociedade, População e Natureza.

Santos, M. F. G., Alves, R. E., \& Roca, M. (2015). Carotenoid composition in oils obtained from palm fruits from the Brazilian Amazon. Grasas y Aceites, 66 (3), e086. http://dx.doi.org/10.3989/gya.1062142

Schiassi, M. C. E. V., Souza, V. R., Lago, A. M. T., Campos, L. G., \& Queiroz, F. (2018). Fruits from the Brazilian Cerrado region: Physico-chemical characterization, bioactive compounds, antioxidant activities, and sensory evaluation. Food Chemistry, 245, 305-311. https://doi.org/10.1016/j.foodchem.2017.10.104

Serra, J. L., Rodrigues, A. M. C., Freitas, R. A., Meirelles, A. J. A., Darnet, S. H., \& Silva, L. H. M. (2019). Alternative sources of oils and fats from Amazonian plants: fatty acids, methyl tocols, total carotenoids and chemical composition. Food Research International, 116, 12-19. https://doi.org/10.1016/j.foodres.2018.12.028

Siqueira, A. P. S., Castro, C. F. S., Silveira, E. V., \& Lourenço, M. F. C. (2016). Chemical quality of Baru almond (Dipteryx alata oil). Ciência Rural, 46 (10), 1865-1867. https://doi.org/10.1590/0103-8478cr20150468

Speranza, P., Falcão, A. O., Macedo, J. A., Silva, L. H. M., Rodrigues, A. M. C., \& Macedo, G. A. (2016). Amazonian Buriti oil: Chemical characterization and antioxidant potential. Grasas y Aceites, 67 (2), e135. https://doi.org/10.3989/gya.0622152

Vieira, J. S. C., Sousa, T. L., Rosas, L. S., Lima, A. L., Ronconi, C. M., \& Mota, C. J. A. (2018). Esterificação e transesterificação homogênea de óleos vegetais contendo alto teor de ácidos graxos livres. Química Nova, 41 (1), 10-16. https://doi.org/10.21577/0100-4042.20170148 